\title{
Anti-proteolytic property and bonding durability of mussel adhesive protein-modified dentine adhesive interface
}

\section{Hui Fang ${ }^{\mathrm{a}}$, Quan-Li Li ${ }^{\mathrm{a}, *}$, Min Han ${ }^{\mathrm{a}}$, May Lei Mei ${ }^{\mathrm{b}}$, Chun Hung Chu ${ }^{\mathrm{b}}$,*}

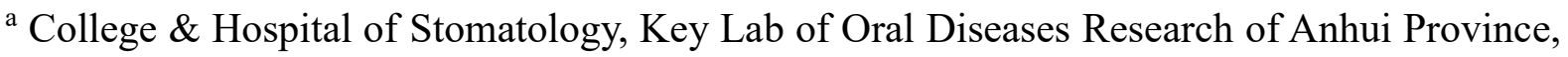
Anhui Medical University, Hefei, 230032, China

${ }^{\mathrm{b}}$ Faculty of Dentistry, The University of Hong Kong, Hong Kong, China

Key words: mussel adhesive protein; collagenase; collagen degradation; dentin adhesion; microtensile bond strength.

\author{
* Corresponding to : $\quad$ Quan-Li Li \\ College \& Hospital of Stomatology \\ Key Lab of Oral Diseases Research of Anhui Province \\ Anhui Medical University \\ Hefei, China
}

Tel: +86551 65118677

Fax : +8655165111538

E-mail: ql-1i@126.com

Chun Hung Chu

Faculty of Dentistry

The University of Hong Kong

Hong Kong, China

Tel: +85228590287

Fax: +85225599013

E-mail: chchu@hku.hk

\section{Abstract}


Resin composite is widely used in dental restoration, but to achieve long-term success of the resin-dentin bond strength is still a challenge due to the degradation of the exposed collagen caused by proteases. This study is to evaluate the influence of mussel adhesive protein (MAP) application on collagenase activity, dentin collagen degradation and microtensile dentin bond strength $(\mu T B S)$. The influence of MAP on collagenase activity was measured by generic colorimetric assay. Thirty human dentin slabs were prepared and allocated into 3 groups $(n=10)$ for application of MAP (experimental group), GM6001 collagenase-inhibitor (GM6001 group, positive control) and distilled water (DW group, negative control). After incubation in collagenase (Clostridiopeptidase-A) solution for 7 days, degradation of dentin collagen matrix was evaluated by measuring hydroxyproline released from the slabs. Another 60 dentin slabs were pretreated by either MAP, GM6001 collagenase-inhibitor or water followed by etch-andrinse adhesive to nano-hybrid composite resin ( $n=20$ in each group). $\mu T B S$ testing was performed before and after thermocycling $\left(\times 2,500\right.$ at $5^{\circ} \mathrm{C}$ and $\left.55^{\circ} \mathrm{C}\right)$ and collagenase challenge $\left(3\right.$ weeks at $\left.37^{\circ} \mathrm{C}\right)$. The morphology of dentin/adhesive interfaces or the fractured interfaces were examined by scanning electron microscopy (SEM). The results demonstrated that MAP significantly inhibited the collagenase activity and was superior to GM6001 collagenase-inhibitor. Fitting of the collagenase inhibition, the result of hydroxyproline concentrations and $\mu T B S s$ (MPa) indicated that MAP efficiently prevented dentin collagen degradation and retarded the deterioration of the dentin bonding of composite restoration over aging. SEM observation showed the fractured dentin surface of MAP group were almost occurred at the top of the hybrid layer, with few collagen fibres observed. Thus, mussel adhesive protein-modified dentine adhesive interface may improve bonding durability in clinical dentistry. 


\section{Introduction}

Resin composite is widely used in dental restoration due to its superior aesthetics and excellent immediate bond strength. ${ }^{1}$ However, achieving long-term success and durability of the resin-dentin bond strength is challenging because of the degradation of the hybrid layer. ${ }^{2,3}$ Hydrolysis of adhesive resin and disorganization of collagen fibrils are the two main factors causing the deterioration of the hybrid layer. ${ }^{4}$ Many etch-and-rinse and self-etch adhesive systems fail to completely infiltrate the demineralized dentin matrix. ${ }^{5}$ Collagen fibrils are exposed with no hydrophobic resin coatings along the bottom of the hybrid layer. These exposed collagen fibrils are vulnerable to degradation by proteases leading to the degeneration of the hybrid layer and the failure of the restoration. The proteases may refer to reactivated endogenous matrix metalloproteinases (MMPs) from dentin ${ }^{6,7}$ and other collagenases from bacteria or saliva ${ }^{8}$.

To sustain the durability of the bond strength, researchers are exploring methods to inhibit protease activity and/or stabilize the ultrastructure of dentin collagen. Nonspecific protease-inhibitors such as chlorhexidine ${ }^{9}$, ethylenediaminetetraacetic acid (EDTA) ${ }^{10}$ and doxycycline $^{11}$ were used. They competitively chelate calcium and zinc ions, which are the necessary cations for the activation of $\mathrm{MMPs}^{5}$ and bacterial collagenases ${ }^{12}$. Although chlorhexidine and EDTA prevent collagen degradation in the hybrid layer, both of them are water-soluble and may dissolve in humid oral environment ${ }^{10,13}$, which limits their long-term effect. Doxycycline could darken the adhesive materials ${ }^{14}$, which affects its aesthetic outcome. Other protease-inhibitors such as glutaraldehyde, riboflavin ${ }^{15}$ and proanthocyanidin ${ }^{16}$ were also used. They inactivate the active sites of proteases and act as cross-linking agents to increase the stiffness of collagen. ${ }^{4}$ However, these proteases also have their limitations. For example, the use of glutaraldehyde has been limited in various applications because of concerns about its cytotoxicity. ${ }^{15}$ The cytotoxicity of riboflavin is still under debate. ${ }^{17}$ In addition, the incorporation of proanthocyanidin into the adhesive was reported to impair the polymerization efficacy of resin. ${ }^{17}$ 
Mussel adhesive protein (MAP), a proteinaceous adhesive secreted by marine mussels, is the key component in the firm attachment of marine mussels to wet surfaces. MAP provides the mussels' resistance to mechanical stresses which arise from tough and adverse marine environments, such as continuous turbulence, tidal waves and temperature swings. ${ }^{18}$ The extraordinary adhesive property of MAP is attributed to the 3,4-dihydroxy-phenylalanine (DOPA) residues. DOPA contains catechol groups that process hydrogen bonding and metalliganding capabilities. ${ }^{19,20}$ Scientists recently developed various DOPA-derivative adhesives ${ }^{21}$, 22 and demonstrated their exceptional properties in dental adhesives ${ }^{23}$ and bone adhesives ${ }^{24}$. However, no study has so far reported on the enzyme-inhibiting properties of MAP. The aim of this study is to evaluate the effects of MAP on collagenase activity, dentine collagen degradation and resin-dentine bond strength. We hypothesized that MAP can (i) inhibit the activity of collagenase, (ii) prevent dentin collagen degradation and (iii) finally improve the durability of the resin-dentin adhesive with the cooperation of MAP use.

\section{Materials and Methods}

\subsection{Materials}

The MAP (recombined mussel adhesive protein, Abcam, Cambridge, UK) used was prepared at a concentration of $1 \mathrm{mg} / \mathrm{mL}$. It was adjusted to a typical marine environmental $\mathrm{pH}$ of 8.5 with $0.1 \mathrm{M}$ sodium hydroxide ${ }^{25}$. The collagenase used was prepared by adding lyophilized powder of Clostridiopeptidase-A in an incubation medium containing zwitterionic organic chemical buffering agent to achieve a concentration of $100 \mathrm{U} / \mathrm{ml}$. The Clostridiopeptidase-A used was high-purity collagenase from Clostridium histolyticum (Type VII, Sigma-Aldrich, St. Louis, MO, USA), and the buffering agent contained $50 \mathrm{mM}$ 4-(2hydroxyethyl)-1-piperazineethanesulfonic acid, $0.36 \mathrm{mM}$ calcium chloride and $0.02 \%$ sodium azide ( $\mathrm{pH}$ 7.4). The collagenase inhibitor used was a synthetic broad spectrum MMP inhibitor (GM6001, Abcam, Cambridge, UK). It was prepared at $20 \mu \mathrm{M}$ concentration with water-based $10 \%(\mathrm{v} / \mathrm{v})$ dimethyl sulphoxide solution. 


\subsection{Inhibition of collagenase activity}

The Sensolyte Generic MMP colorimetric assay kit (AnaSpec Inc, Fremont, CA, USA) was used for high throughput screening of the MMP's inhibitors. It contains thiopeptolide, which can be cleaved by MMPs or collagenases to release a sulfhydryl group. The sulfhydryl group reacts with 5, 5'-dithiobis (2-nitrobenzoic acid) to produce 2-nitro-5-thiobenzoic acid. ${ }^{26}$ This acid is a coloured-reaction product which can be detected spectrophotometrically at 412 nm. ${ }^{26}$ This assay was performed in a 96-well plate with five replicate wells for each group. Fifteen samples were divided into the three groups $(n=5)$. The first group was the experimental (MAP) group (10 $\mu \mathrm{L}$ of MAP and $40 \mu \mathrm{L}$ of Clostridiopeptidase-A). The second group was the positive control (GM6001) group with collagenase-inhibitor (10 $\mu \mathrm{L}$ of GM6001 and $40 \mu \mathrm{L}$ of Clostridiopeptidase-A). The third group was a negative control (DW) group with distilled water (10 $\mu \mathrm{L}$ of distilled water and $40 \mu \mathrm{L}$ of Clostridiopeptidase-A). All reagents were mixed well in each experimental well and pre-incubated for 20 min to avoid the burst of collagenase (MMP9) activity that may occur when all reagents are mixed together simultaneously. After preincubation, $50 \mu \mathrm{L}$ of the thiopeptolide (Sensolyte Generic MMP colorimetric assay kit) was added to reach a total of $100 \mu \mathrm{L}$ for each well. The reagents were mixed thoroughly for $30 \mathrm{~s}$. The absorbance at $412 \mathrm{~nm}$ was read by a microplate spectrophotometer ( $\mu$ Quant, BioTek, Winooski, VT, USA) at $10 \mathrm{~min}$ intervals for $60 \mathrm{~min}$. Glutathione reference standard containing $0 \sim 200 \mu \mathrm{M}$ of reduced glutathione was prepared. Reduced glutathione is a tripeptide (gglutamylcysteinylglycine) with a free thiol group, which reacts with 5, 5'-dithiobis (2nitrobenzoic acid) and produces a yellow colour. Reduced glutathione can be used as a reference standard to calculate the concentration of sulfhydryl groups in the sample. The collagenase activity under the function of collagenase inhibitor was calculated as:

$$
\text { Collagenase activity }=\frac{\mu \mathrm{M}(\mathrm{SH}) \times \mathrm{r}}{\mathrm{mg} / \mathrm{mL}(\mathrm{Col}) \times \mathrm{t}}
$$

where $\mu \mathrm{M}(\mathrm{SH})$ and $\mathrm{mg} / \mathrm{ml}(\mathrm{Col})$ are the concentration of sulfhydryl groups $(\mu \mathrm{mol} / \mathrm{L})$ and bacterial collagenase $(\mathrm{mg} / \mathrm{mL}), \mathrm{r}$ represents the dilution ratio of test sample, and t represents reaction time. 


\subsection{Inhibiting the degradation of demineralized dentin matrix}

\subsubsection{Dentine specimen preparation}

Thirty sound human third molars were collected from patients requiring extraction with informed consent. The research protocol was approved by the local Independent Ethics Committee. The teeth were stored at $4{ }^{\circ} \mathrm{C}$ in $0.02 \%$ sodium azide to prevent bacterial growth and used within one month of extraction. Thirty dentin slabs $(5 \mathrm{~mm} \times 5 \mathrm{~mm} \times 1.5 \mathrm{~mm})$ were prepared and polished with a 600-grit silicon carbide paper under running water to create a smooth surface. The slabs were cleaned using detergent solution, acetone, ethanol and distilled water. They were etched with $37 \%$ phosphoric acid for 60 s and then rinsed thoroughly with distilled water. The etched slabs were randomly divided into MAP, GM6001 and DW group and incubated at $37^{\circ} \mathrm{C}$ for $5 \mathrm{~min}$ in a polypropylene tube containing $20 \mu \mathrm{L}$ of MAP, GM6001 collagenase-inhibitor and distilled water, respectively. Then, $800 \mu \mathrm{L}$ collagenase Clostridiopeptidase-A were added into each polypropylene tube. The tubes were sealed and incubated in a shaker-water bath $(60$ cycles $/ \mathrm{min})$ at $37^{\circ} \mathrm{C}$ for 7 days.

\subsubsection{Determination of degraded collagen}

The degradation of collagen was determined by estimating hydroxyproline (HYP), an amino acid characteristic of collagen. ${ }^{27}$ After 7 days of incubation, $400 \mu \mathrm{L}$ of the medium was collected from each vial and transferred to a labelled polypropylene tube. An equal volume of $6 \mathrm{M}$ hydrochloric acid was added into the tube, mixed and hydrolysed at $98^{\circ} \mathrm{C}$ for $24 \mathrm{~h} .{ }^{28} \mathrm{After}$ cooling, the hydrolysates were lyophilized and re-solubilized in $600 \mu \mathrm{L}$ of distilled water. To quantitatively analyse HYP, each sample was determined in triplicate, as described previously ${ }^{29}$, with slight modifications. Briefly, 0.056 M chloramine-T reagent was mixed into the above hydrolysate, and then oxidation was allowed to proceed at room temperature. After a 25-min reaction, $1 \mathrm{M}$ Ehrlich's reagent was added to react with the oxidation product, which finally generated the chromophore. The absorbance was measured by a spectrophotometer ( $\mu$ Quant, BioTek) at $550 \mathrm{~nm}$. Simultaneously, a HYP standard solution containing $0-50 \mu \mathrm{g} / \mathrm{mL}$ of hydroxyproline was prepared. The standard curve had a coefficient of determination of 0.98 . 


\subsection{Microtensile bond strength test}

Another 60 dentine slabs were prepared from sound human third molars. They were etched with $37 \%$ phosphoric acid for 15s and randomly divided into MAP, GM6001 and DW group. In MAP group, the dentin surface was scrubbed with a microbrush containing MAP for 60s. For the GM6001 and DW group, the dentin surface was scrubbed with GM6001 collagenase-inhibitor and distilled water, respectively. Then, the dentin surface was treated with an ethanol-based, single-component adhesive resin (Gluma Comfort Bond, Heraeus Kulzer, Hanau, Germany) which was light-cured according to the manufacturer's instructions. A universal nano-hybrid composite resin (Tetric EvoCeram, Ivoclar Vivadent, Schaan, Liechtenstein) of $6 \mathrm{~mm}$ was built up incrementally. The composite resin at $1 \mathrm{~mm}$ increment was polymerized for 20s using an LED device (Bluephase 20i, IvoclareVivadent, Schaan, Liechtenstein). The composite bonded dentin slabs were stored in distilled water at $37^{\circ} \mathrm{C}$ for 24h. They were longitudinally sectioned into beams with a cross-sectional dimension of 0.8 $\times 0.8 \mathrm{~mm}^{2}$ for microtensile bond strength ( $\left.\mu \mathrm{TBS}\right) \mathrm{test}^{30}$. Each group was further divided into two subgroups, and a total of 140 beams were created from 20 slabs. The beams in the first subgroup were immediately subjected to $\mu$ TBS test. The beams in the second subgroup received thermocycling and collagenase challenge before the $\mu$ TBS test. Thermocycling was performed for 2,500 cycles by putting the beams between $5^{\circ} \mathrm{C}$ and $55^{\circ} \mathrm{C}$ with a dwell time of $15 \mathrm{~s}$. The beams were stored in the collagenase solutions at $37^{\circ} \mathrm{C}$ for 3 weeks. The collagenase solution was refreshed every week. The $\mu$ TBS test was performed with a digital universal testing machine (WDS-20, Jinan Fangyuan Co., Shandong, China) at a crosshead speed of 1 $\mathrm{mm} / \mathrm{min}$ until failure. The dimension of the fracture area was recorded using a digital caliper to the nearest $0.01 \mathrm{~mm}$, and $\mu \mathrm{TBS}$ value (in $\mathrm{MPa}$ ) was calculated.

Field emission scanning electron microscopy (FE-SEM; Sirion 200, FEI Co, Hillsboro, OR, USA) was used to study the morphology of the dentin/adhesive interfaces of the specimens before $\mu$ TBS testing and the morphology of the fractured dentin interfaces of the fractured samples after $\mu$ TBS testing. 


\subsection{Statistical analysis}

Kolmogorov-Smirnov test was used to test the normality of the data. Levene test was used to test whether the samples had equal variances. One-way ANOVA was used to analyse the amount of hydroxyproline released from the dentin. Tukey's test was used for multiple comparisons. Kruskal-Wallis test with Dunn's multiple comparison was used to compare $\mu$ TBS between groups for each time point. Wilcoxon rank sum test was used to analyse the difference between the immediate and aging within each group. The analyses were performed using IBM SPSS Version 20.0 software (IBM Corporation, Armonk, New York, USA). The cutoff level for significance was taken as $5 \%$ for all of the analyses.

\section{Results}

The mean $( \pm \mathrm{SD})$ collagenase activity $(\mathrm{nmol} / \mathrm{min} / \mathrm{mg})$ for the MAP, GM6001 and DW group were $20.22 \pm 0.93,29.11 \pm 1.17$ and $31.39 \pm 0.52(p<0.01$, MAP $<$ GM6001 < DW $)$. A similar trend was observed for the measurement of hydroxyproline concentration. The mean hydroxyproline concentration $(\mu \mathrm{g} / \mathrm{mL})$ for the MAP, GM6001 and DW group were $2.70 \pm$ $0.53,4.00 \pm 1.19$ and $5.40 \pm 1.00$, respectively $(p<0.01$, MAP $<$ GM6001 $<$ DW).

The mean $( \pm \mathrm{SD})$ values $(\mathrm{MPa})$ of the immediate $\mu$ TBS test for the MAP, GM6001 and DW group were $17.47 \pm 5.98,18.45 \pm 6.52$ and $17.31 \pm 4.53$, respectively $(p=0.624)$. After thermocycling and exposure to collagenase challenge, the mean $\mu$ TBS values (MPa) for the MAP, GM6001 and DW group were $11.39 \pm 2.52,10.77 \pm 3.16$ and $5.83 \pm 2.02 \mathrm{MPa}(p<$ 0.001, (MAP, GM6001 > DW).

Scanning electron micrographs revealed different morphology at the bonding interface among the three groups (Fig. 1). The bonding interfaces detected before the immediate $\mu$ TBS test showed that the adhesive resin infiltrated into the collagen matrix and formed resin tags to occlude the dentinal tubules, and the intact hybrid layer bonded tightly between the dentin and resin (Figs.1a, 1c and 1e). In the MAP group, collagen fibres were hardly identified (Fig 1a), while a few fibres were observed in the GM6001 and DW group (Figs. 1c and 1e). This might 
be attributed to the extensive coated MAP on collagen surfaces or MAP cross-linked collagen fibres, which induced the complete infiltration of resin monomer. After thermocycling and collagenase challenge, the integrity of the bonding interfaces was broken. Numerous microfractures were generated in the hybrid zone, and collagen fibres were exposed among all groups (Figs.1b, 1d and 1f). In the MAP (Fig.1b) and GM6001 (Fig.1d) group, the collagen fibres had slighter degradation especially for the MAP group. In the DW group, on the other hand, larger spaces existed between the resin tags and dentinal tubules, suggesting that more collagen fibres had degraded (Fig.1f). The representative morphology of the interfaces corresponded to the $\mu$ TBS results.

Scanning electron micrographs of the fractured dentin surface among the three tested groups are reflected in Figure 2 and 3. In the immediate $\mu$ TBS test groups, modes of failure were mainly mixed, involving adhesive resin or resin composite (Figs. 2). Concretely, in the MAP and GM6001 group, fractures frequently occurred at the top of the hybrid layer, leaving the underlying dentin covered by adhesives (Figs.2a and 2b, 2c and 2d). However, in the DW group, failures occurred in the middle of the hybrid layer with numerous resin tags tightly filling the dentinal tubules, and a few collagen fibres could be observed (Figs.2e and 2f). For the $\mu$ TBS tests after thermocycling and collagenase challenge, the fractured dentin surfaces mostly occurred in the hybrid layer (Fig.3). In the MAP group, failures occurred the top of the hybrid layer, and the degradation of collagen fibres was hardly identified (Figs. 3a and 3b). In contrast, fractures in the DW group occurred at the bottom of the hybrid layer and even near to the dentin, showing a large degree of degradation of collagen fibres. Meanwhile, numerous dentinal tubules were empty, with resin tags having been pulled out (Figs.3e and 3f). Fractures in the GM6001 group mostly appeared at the top of the hybrid layer and partially occurred at the bottom of the hybrid layer with less degradation of collagen fibres (Figs. 3c and 3d).

\section{Discussion}

Degradation of collagen fibres beneath the hybrid layer is one of the main reasons for the long-term failure in resin-dentin bond restorations. ${ }^{4,5}$ The present study demonstrated that 
MAP had inhibitory effects, which was more effective than a synthetic broad spectrum MMP inhibitor against bacterial collagenase activity and protected dentin collagen from degradation.

GM6001 is a synthetic broad spectrum MMP inhibitor which has a collagen-like structure that binds to the active site of MMPs. In addition, it has a hydroxamate structure to chelate the zinc ions and inhibit the proteolytic activities of MMP-1, $-2,-8$ and $-9^{31,32}$. GM6001 is 100 times more potent than chlorhexidine in the inhibition of collagenase activities. ${ }^{31}$ In this study, MAP exhibited greater potency to inhibit bacterial collagenase activity and prevent collagen degradation. Clostridium histolyticum collagenase is a bacterial collagenase which belongs to the M9 metalloproteinase family ${ }^{33}$ and has been used in laboratory studies to examine the effect of collagenase inhibitors on collagenase-induced dentin collagen degradation $28,34,35$. Thus, Clostridium histolyticum collagenase was chosen as the collagenase model to degrade the exposed collagen fibres. It has four segments, one of which incorporates the catalytic zinc domain and calcium binding sites ${ }^{33,36}$, showing a similar action mechanism as $\mathrm{MMPs}^{37}$. However, compared with vertebrate MMPs, which cleave the collagen into 1/4 and 3/4 fragments at a single site, bacterial collagenases can hydrolyse triple-helical collagens at multiple sites, resulting in more small peptides for HYP assay ${ }^{27,28}$.

MAPs are polyphenolic proteins contributing to underwater adhesion by marine mussels. ${ }^{18}$ DOPA can be found in all types of MAPs and provide the most dominant influence upon the durable and water-impervious adhesive strength. ${ }^{21}$ The commercially available MAPs are obtained using protein extraction techniques or recombinant protein techniques. Due to difficulties in extracting sufficient natural MAPs and inconsistencies in their chemical purities $^{18}$, recombinant MAP was used in this study. The recombinant MAP herein belong to one type of MAP family, produced by the genomic sequence of Mytilus edulis foot protein 1 (Mefp-1). Distinguished by the fact that mussels' extraordinary adhesion strength can be attributed to the cooperation of various byssus proteins such as Mefp-2, 3, 4, 5, 6 ${ }^{38,39}$, the adhesion strength of recombinant MAP may be limited. This perhaps explains why the usage of recombinant MAP caused no significant increase in the immediate $\mu$ TBS values. 
To explain the effective inhibition of bacterial collagenase by MAP, we speculated that the catechol group in DOPA may perform a dominant function. With the strong metal-chelation ability of the catechol group, MAP may competitively chelate $\mathrm{Ca}^{2+}$, the essential element for the action of bacterial collagenase, subsequently inducing collagenase inhibition. A similar chelation inhibitory mechanism was proposed in the experiment involving EDTA ${ }^{10}$. As a divalent metal chelator, EDTA can inhibit MMP activity by chelating the zinc ions in the catalytic domain of MMPs and/or by removing calcium ions from the three-dimensional collagen structure ${ }^{10,40}$. The inhibitory mechanism of MAP on bacterial collagenase may be similar to that of EDTA, but the effect of MAP against various human MMPs still needed further studies. Additionally, as a potent cross-linker, MAP may cross-link with collagen fibres through covalent bonds between catechol groups of MAP and amino groups of collagen fibres. Cross-linking agents can render collagen stiff by preventing its triple-helix conformation from uncoiling $^{41}$. As a result, it enhances the resistance of cross-linked collagen in response to degradation by collagenase. Cross-linking agents can also cross-link active and allosteric enzyme sites which directly interfere with their activity ${ }^{41}$. A study demonstrated that proanthocyanidin (a typical cross-linker) significantly reduced the release of HYP from demineralized dentin beams and increased resin-dentin bond strength ${ }^{16}$.

A bonding model with an acid-etched and rinse adhesive system was used to evaluate the effect of MAP on resin-dentin bonding strength and durability. The process of acid etching exposed the collagen in order to facilitate observation of the effect of MAP. Resin-dentin bonding strength and its durability are known to depend not only on the inhibition of exposed collagen degradation but also on the amount of moisture on the dentin surfaces. Water generally prohibits resin from bonding to dentin. MAPs have gained considerable interest because of their superior water-resistant adhesive ability. Research has emphasized that the DOPA-rich MAPs may act as adhesive primers, overcoming repulsive hydration forces by displacing surface-bond water and generating strong surface adhesion ${ }^{42}$. Meanwhile, several musselmimetic adhesives with superior bonding strength have been successfully synthesized ${ }^{21,23,24}$. Therefore, we propose that MAPs will improve the 'wetting bond' of resin-dentin bonding strength. With this goal in mind, we pretreated the demineralized dentin surface with $1 \mathrm{mg} / \mathrm{mL}$ 
MAP. We inferred from the special bonding characteristic of natural MAPs that a significantly higher $\mu$ TBS should be observed in the MAP group; however, the result did not agree with our inference. The immediate $\mu$ TBS of the MAP group was approximate to that of the DW group and the GM6001-treated group. We speculated that the result may be attributed to the lower MAP concentration or the shorter time of MAP pretreatment. Regarding the time of pretreating the collagen matrix with several agents, the treatment of demineralized dentin with 3.75 mass \% PA for 5, 15 or 30s reportedly yielded sufficient cross-linking formation to protect the dentin matrix from degradation ${ }^{43}$; in addition, the GM6001-treatment for $30 \mathrm{~s}$ significantly decreased bond degradation after 1 year ${ }^{31}$. Thus, in this study, on the basis of feasibility in clinical practice, we pretreated the resin-dentin bond specimens with MAP or GM6001 for 1 min. However, the optimal concentration and time for application of MAP still require further study.

To evaluate the ability of the bonding interface to resist the collagenase, we exposed the specimens to thermocycling for 2500 cycles (from $5^{\circ} \mathrm{C}$ to $55^{\circ} \mathrm{C}$ with a dwell time of $15 \mathrm{~s}$ in each temperature) prior to subjecting them to a $100 \mathrm{U} / \mathrm{mL}$ bacterial collagenase solution for 3 weeks at $37^{\circ} \mathrm{C}$. Thermocycling functions by partially breaking down the bonding interface to facilitate the interaction between collagenase and collagen in the hybrid zone. The results show that the durability of the adhesive was improved when MAP was applied to the collagen matrix at the bonding interface. Besides this, numerous methods, such as the application of collagen cross-linkers during adhesive restorative procedures ${ }^{44}$, ethanol-wet bonding technique ${ }^{45}$ and the use of MMP inhibitors ${ }^{46}$, have been proposed to improve dentin bond strength and durability. For example, an MAP-inspired catechol-functionalized synthetic polymer as a dental adhesive can reportedly improve the bond strength between the contaminated dentin surface and the composite restoration ${ }^{23}$. Here, we found that MAP could improve bond durability. The morphology of bonding interfaces also indirectly supported the $\mu$ TBS results.

\section{Conclusion}

In this laboratory study, MAP inhibited collagenase activity and protected the 
demineralized dentin collagen matrix from degradation. Demineralized dentin collagen matrix treated with MAP enhanced the resistance of the bonding interface to enzymatic degradation. This novel protocol may be used to improve bonding durability in clinical dentistry.

\section{Acknowledgement}

This study was supported by NSFC/RGC Joint Research Scheme (No.8106116051and1N_HKU 776/10) and NSFC (No.81571018). 


\section{References}

(1) Inoue, S.; Vargas, M. A.; Abe, Y.; Yoshida, Y.; Lambrechts, P.; Vanherle, G.; Sano, H.; Van Meerbeek, B. Microtensile Bond Strength of Eleven Modern Adhesives to Dentin. $J$. Adhes. Dent. 2001, 3, 237-246.

(2) Hashimoto, M.; Ohno, H.; Sano, H.; Kaga, M.; Oguchi, H. Degradation Patterns of Different Adhesives and Bonding Procedures. J. Biomed. Mater. Res., Part B. 2003, 66, $324-330$.

(3) Hashimoto, M.; Fujita, S.; Kaga, M.; Yawaka, Y. In Vitro Durability of One-bottle Resin Adhesives Bonded to Dentin. Dent. Mater. J. 2007, 26, 677-686.

(4) Frassetto, A.; Breschi, L.; Turco, G.; Marchesi, G.; Di Lenarda, R.; Tay, F. R.; Pashley, D. H.; Cadenaro, M. Mechanisms of Degradation of the Hybrid Layer in Adhesive Dentistry and Therapeutic Agents to Improve Bond Durability-A Literature Review. Dent. Mater. 2016, 32, e41-53.

(5) Tjäderhane, L.; Nascimento, F. D.; Breschi, L.; Mazzoni, A.; Tersariol, I. L.; Geraldeli, S.; Tezvergil-Mutluay, A.; Carrilho, M. R.; Carvalho, R. M.; Tay, F. R. Optimizing Dentin Bond Durability: Control of Collagen Degradation by Matrix Metalloproteinases and Cysteine Cathepsins. Dent. Mater. 2013, 29, 116-135.

(6) Mazzoni, A.; Pashley, D. H.; Nishitani, Y.; Breschi, L.; Mannello, F.; Tjäderhane, L.; Toledano, M.; Pashley, E. L.; Tay, F. R. Reactivation of Inactivated Endogenous Proteolytic Activities in Phosphoric Acid-etched Dentine by Etch-and-rinse Adhesives. Biomaterials 2006, 27, 4470-4476.

(7) Lehmann, N.; Debret, R.; Roméas, A.; Magloire, H.; Degrange, M.; Bleicher, F.; Sommer, P.; Seux, D. Self-etching Increases Matrix Metalloproteinase Expression in the Dentin-pulp Complex. J. Dent. Res. 2009, 88, 77-82.

(8) Ferrari, M.; Mason, P.; Goracci, C.; Pashley, D. H.; Tay, F. Collagen Degradation in Endodontically Treated Teeth after Clinical Function. J. Dent. Res. 2004, 83, 414-419.

(9) Gendron, R.; Grenier, D.; Sorsa, T.; Mayrand, D. Inhibition of The Activities of Matrix Metalloproteinases 2, 8, and 9 by Chlorhexidine. Clin. Diagn. Lab. Immunol. 1999, 6, $437-$ 439. 
(10) Thompson, J. M.; Agee, K.; Sidow, S. J.; McNally, K.; Lindsey, K.; Borke, J.; Elsalanty, M.; Tay, F. R.; Pashley, D. H. Inhibition of Endogenous Dentin Matrix Metalloproteinases by Ethylenediaminetetraacetic Acid. J. Endodontics 2012, 38, 62-65.

(11) Horwitz, V.; Dachir, S.; Cohen, M.; Gutman, H.; Cohen, L.; Fishbine, E.; Brandeis, R.; Turetz, J.; Amir, A.; Gore, A. The Beneficial Effects of Doxycycline, an Inhibitor of Matrix Metalloproteinases, on Sulfur Mustard-induced Ocular Pathologies Depend on the Injury Stage. Curr. Eye Res. 2014, 39, 803-812.

(12) Eckhard, U.; Schönauer, E.; Brandstetter, H. Structural Basis for Activity Regulation and Substrate Preference of Clostridial Collagenases G, H, and T. J. Biol. Chem. 2013, 288, 20184-20194.

(13) Sabatini, C.; Patel, S. K. Matrix Metalloproteinase Inhibitory Properties of Benzalkonium Chloride Stabilizes Adhesive Interfaces. Eur. J. Oral Sci. 2013, 121, 610-616.

(14) Feitosa, S.; Palasuk, J.; Kamocki, K.; Geraldeli, S.; Gregory, R. L.; Platt, J.; Windsor, L.; Bottino, M. C. Doxycycline-encapsulated Nanotube-modified Dentin Adhesives. J. Dent. Res. 2014, 93, 1270-1276.

(15) Seseogullari-Dirihan, R.; Mutluay, M.; Vallittu, P.; Pashley, D. H.; Tezvergil-Mutluay, A. Effect of Pretreatment with Collagen Crosslinkers on Dentin Protease Activity. Dent. Mater. 2015, 31, 941-947.

(16) Epasinghe, D. J.; Yiu, C. K. Y.; Burrow, M. F.; Hiraishi, N.; Tay, F. R. The Inhibitory Effect of Proanthocyanidin on Soluble and Collagen-bound Proteases. J. Dent. 2013, 41, 832-839.

(17) Hass, V.; Luque-Martinez, I. V.; Gutierrez, M. F.; Moreira, C. G.; Gotti, V. B.; Feitosa, V. P.; Koller, G.; Otuki, M. F.; Loguercio, A. D.; Reis, A. Collagen Cross-linkers on Dentin Bonding: Stability of the Adhesive Interfaces, Degree of Conversion of the Adhesive, Cytotoxicity and in situ MMP Inhibition. Dent. Mater. 2016, 32, 732-741.

(18) Silverman, H. G.; Roberto, F. F. Understanding Marine Mussel Adhesion. Mar. Biotechnol. 2007, 9, 661-681.

(19) Lee, H.; Scherer, N. F.; Messersmith, P. B. Single-molecule Mechanics of Mussel Adhesion. Proc. Natl. Acad. Sci. U. S. A. 2006, 103, 12999-13003. 
(20) Monahan, J.; Wilker, J. J. Specificity of Metal Ion Cross-linking in Marine Mussel Adhesives. Chem. Commun. 2003, 14, 1672-1673.

(21) Kim, B. J.; Oh, D. X.; Kim, S.; Seo, J. H.; Hwang, D. S.; Masic, A.; Han, D. K.; Cha, H. J. Mussel-mimetic Protein-based Adhesive Hydrogel. Biomacromolecules 2014, 15, 15791585.

(22) Zhou, J.; Defante, A. P.; Lin, F.; Xu, Y.; Yu, J.; Gao, Y.; Childers, E.; Dhinojwala, A.; Becker, M. L. Adhesion Properties of Catechol-based Biodegradable Amino Acid-based Poly (ester urea) Copolymers Inspired from Mussel Proteins. Biomacromolecules 2014, 16, 266-274.

(23) Lee, S.-B.; González-Cabezas, C.; Kim, K.-M.; Kim, K.-N.; Kuroda, K. CatecholFunctionalized Synthetic Polymer as a Dental Adhesive to Contaminated Dentin Surface for a Composite Restoration. Biomacromolecules 2015, 16, 2265-2275.

(24) Nordberg, A.; Antoni, P.; Montanez, M. I.; Hult, A.; Von Holst, H.; Malkoch, M. Highly Adhesive Phenolic Compounds as Interfacial Primers for Bone Fracture Fixations. ACS Appl. Mater. Interfaces 2010, 2, 654-657.

(25) Kim, S.; Park, C. B. Dopamine-induced Mineralization of Calcium Carbonate Vaterite Microspheres. Langmuir 2010, 26, 14730-14736.

(26) Tezvergil-Mutluay, A.; Agee, K.; Uchiyama, T.; Imazato, S.; Mutluay, M.; Cadenaro, M.; Breschi, L.; Nishitani, Y.; Tay, F.; Pashley, D. H. The Inhibitory Effects of Quaternary Ammonium Methacrylates on Soluble and Matrix-bound MMPs. J. Dent. Res. 2011, 90, $535-540$.

(27) Mei, M. L.; Ito, L.; Cao, Y.; Li, Q. L.; Lo, E. C.; Chu, C. H. Inhibitory Effect of Silver Diamine Fluoride on Dentine Demineralisation and Collagen Degradation. J. Dent. Res. 2013, 41, 809-817.

(28) Kato, M. T.; Leite, A. L.; Hannas, A. R.; Calabria, M. P.; Magalhaes, A. C.; Pereira, J. C.; Buzalaf, M. A. Impact of Protease Inhibitors on Dentin Matrix Degradation by Collagenase. J. Dent. Res. 2012, 91, 1119-1123.

(29) Reddy, G. K.; Enwemeka, C. S. A Simplified Method for the Analysis of Hydroxyproline in Biological Tissues. Clin. Biochem. 1996, 29, 225-229.

(30) Sezinando, A.; Luque-Martinez, I.; Munoz, M. A.; Reis, A.; Loguercio, A. D.; Perdigao, 
J. Influence of a Hydrophobic Resin Coating on the Immediate and 6-month Dentin Bonding of Three Universal Adhesives. Dent. Mater. 2015, e236-246.

(31) Breschi, L.; Martin, P.; Mazzoni, A.; Nato, F.; Carrilho, M.; Tjäderhane, L.; Visintini, E.; Cadenaro, M.; Tay, F. R.; Dorigo, E. D. S. Use of a Specific MMP-inhibitor (galardin) for Preservation of Hybrid Layer. Dent. Mater. 2010, 26, 571-578.

(32) Galardy, R. E.; Cassabonne, M. E.; Giese, C.; Gilbert, J. H.; Lapierre, F.; Lopez, H.; Schaefer, M. E.; Stack, R.; Sullivan, M.; Summers, B.; et al. Low Molecular Weight Inhibitors in Corneal Ulcerationa. Ann. N. Y. Acad. Sci. 1994, 732, 315-323.

(33) Scozzafava, A.; Supuran, C. T. Protease Inhibitors: Synthesis of Clostridium Histolyticum Collagenase Inhibitors Incorporating Sulfonyl-L-alanine Hydroxamate Moieties. Bioorg. Med. Chem. Lett. 2000, 10, 499-502.

(34) Kishen, A.; Shrestha, S.; Shrestha, A.; Cheng, C.; Goh, C. Characterizing the Collagen Stabilizing Effect of Crosslinked Chitosan Nanoparticles Against Collagenase Degradation. Dent. Mater. 2016, 32, 968-977.

(35) Pavan, M.; Galesso, D.; Secchieri, C.; Guarise, C. Hyaluronic Acid Alkyl Derivative: A Novel Inhibitor of Metalloproteases and Hyaluronidases. Int. J. Biol. Macromol. 2016, 84, 221-226.

(36) Eckhard, U.; Schönauer, E.; Brandstetter, H. Structural Basis for Activity Regulation and Substrate Preference of Clostridial Collagenases G, H, and T. J. Biol. Chem. 2013, 288, 20184-20194.

(37) Kumar D.; Gupta S.P. A Quantitative Structure-activity Relationship Study on Some Matrix Metalloproteinase and Cllagenase Ihibitors. Bioorg. Med. Chem. 2003, 11, 421-426.

(38) Lee, B. P.; Messersmith, P. B.; Israelachvili, J. N.; Waite, J. H. Mussel-inspired Adhesives and Coatings. Annu. Rev. Mater. Res. 2011, 41, 99-132.

(39) Jenkins, C. L.; Meredith, H. J.; Wilker, J. J. Molecular Weight Effects upon the Adhesive Bonding of a Mussel Mimetic Polymer. ACS Appl. Mater. Interfaces 2013, 5, 5091-5096. (40) Tezvergil-Mutluay, A.; Agee, K. A.; Hoshika, T.; Carrilho, M.; Breschi, L.; Tjaderhane, L.; Nishitani, Y.; Carvalho, R. M.; Looney, S.; Tay, F. R.; Pashley, D. H. The Requirement of Zinc and Calcium ions for Functional MMP Activity in Demineralized Dentin Matrices. Dent. Mater. 2010, 26, 1059-1067. 
(41) Sabatini, C.; Patel, S. K. Mechanisms Regulating the Degradation of Dentin Matrices by Endogenous Dentin Proteases and their Role in Dental Adhesion. A Review. Amer. J. Dent. 2014, 27, 203-214.

(42) Petrone, L.; Kumar, A.; Sutanto, C. N.; Patil, N. J.; Kannan, S.; Palaniappan, A.; Amini, S.; Zappone, B.; Verma, C.; Miserez, A. Mussel Adhesion is Dictated by Time-regulated Secretion and Molecular Conformation of Mussel Adhesive Proteins. Nat. Commun. 2015. DOI: $10.1038 /$ ncomms 9737.

(43) Liu, Y.; Dusevich, V.; Wang, Y. Proanthocyanidins Rapidly Stabilize the Demineralized Dentin Layer. J. Dent. Res. 2013, 92, 746-752

(44) Hechler, B.; Yao, X.; Wang, Y. Proanthocyanidins Alter Adhesive/dentin Bonding Strengths when included in a Bonding System. Amer. J Dent. 2012, 25, 276-280.

(45) Yang, H.; Guo, J.; Deng, D.; Chen, Z.; Huang, C. Effect of Adjunctive Application of Epigallocatechin-3-gallate and Ethanol-wet Bonding on Adhesive-dentin Bonds. J. Dent. 2016, 44, 44-49.

(46) De Munck, J.; Van den Steen, P. E.; Mine, A.; Van Landuyt, K. L.; Poitevin, A.; Opdenakker, G.; Van Meerbeek, B. Inhibition of Enzymatic Degradation of Adhesivedentin Interfaces. J. Dent. Res. 2009, 88, 1101-1106. 


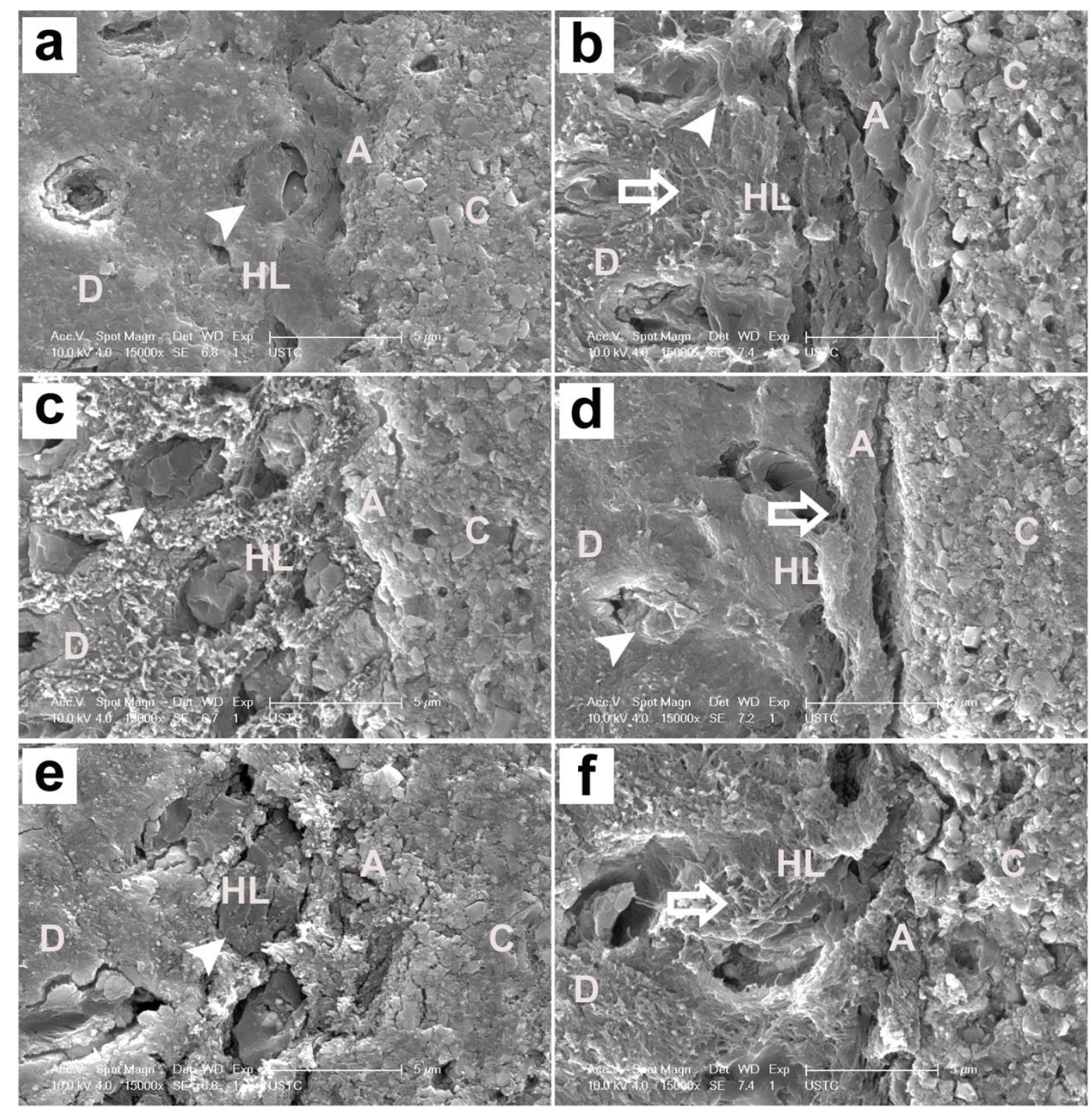

Figure 1. SEM micrographs $(\times 15000)$ of resin-dentin bonding interface among the three tested groups. (a) MAP group detected immediately; (b) MAP group detected after thermocycling and collagenase challenge; (c) GM6001 group detected immediately; (d) GM6001 group detected after thermocycling and collagenase challenge; (e) DW group detected immediately; (f) DW group detected after thermocycling and collagenase challenge. D: dentin, HL: hybrid layer, A: adhesive, C: composite resin. White solid arrows: dentinal tubules with resin tags; White line arrows: exposed collagen fibres. 


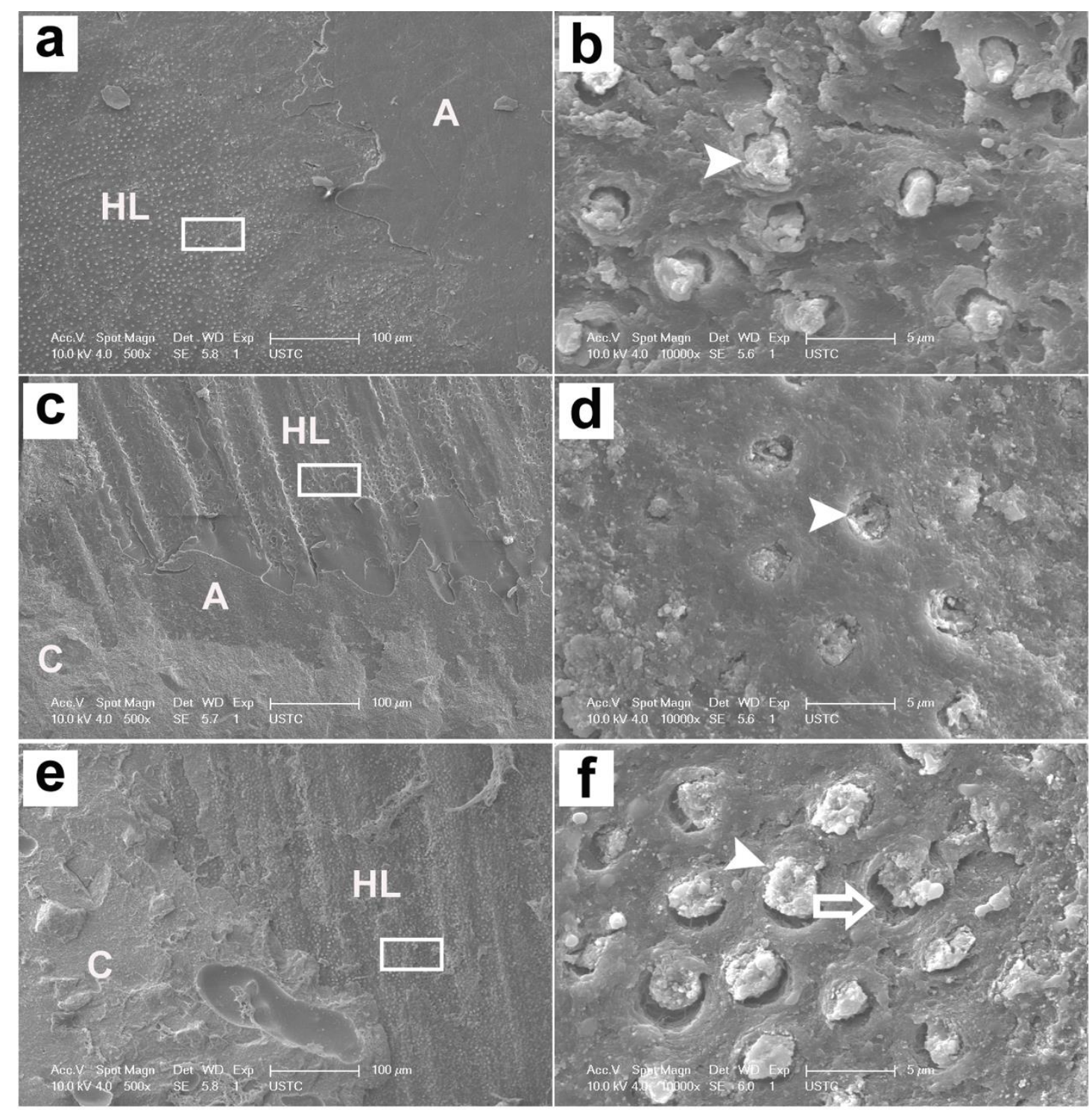

Figure 2. SEM micrographs of fractured dentin surface among the three tested groups with immediate microtensile bond strength tests. (a) $500 \times$ magnification view of MAP group; (b) $10000 \times$ magnification view of the area limited by the rectangle in panes (a); (c) $500 \times$ magnification view of the GM6001 group; (d) $10000 \times$ magnification view of the area limited by the rectangle in panes (c); (e) $500 \times$ magnification view of the DW group;

(f) $10000 \times$ magnification view of the area limited by the rectangle in panes (e). HL: hybrid layer, A: adhesive, C: composite resin. White solid arrows: dentinal tubules with resin tags; White line arrows: exposed collagen fibres. 


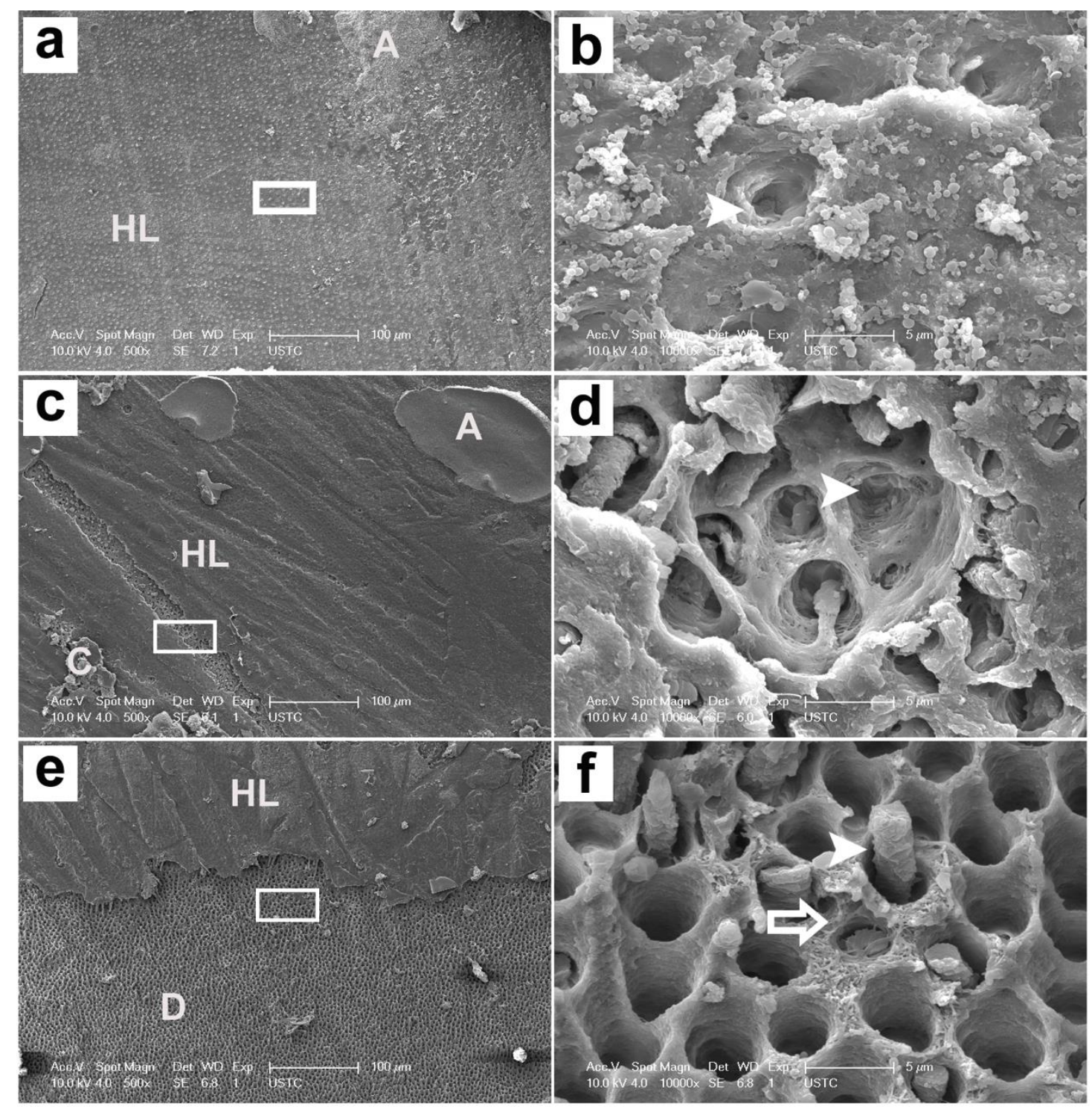

Figure 3. SEM micrographs of fractured dentin surface among the three tested groups with microtensile bond strength tests after thermocycling and collagenase challenge. (a) $500 \times$ magnification view of the MAP group; (b) $10000 \times$ magnification view of the area limited by the rectangle in panes (a); (c) $500 \times$ magnification view of the GM6001 group; (d) $10000 \times$ magnification view of the area limited by the rectangle in panes (c); (e) $500 \times$ magnification view of the DW group; (f) $10000 \times$ magnification view of the area limited by the rectangle in panes (e). HL: hybrid layer, A: adhesive, C: composite resin. White solid arrows: dentinal tubules with resin tags; White line arrows: exposed collagen fibres. 
Table of Contents Graphic

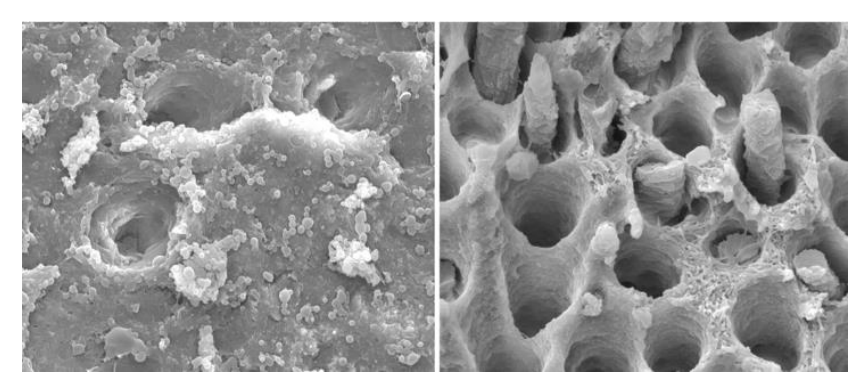

